\title{
Direct numerical simulation of the near-field dynamics of annular gas-liquid two-phase jets
}

\author{
George A. Siamas, Xi Jiang, ${ }^{\text {a) }}$ and Luiz C. Wrobel \\ Department of Mechanical Engineering, School of Engineering and Design, Brunel University, \\ Uxbridge UB8 3PH, United Kingdom
}

(Received 15 May 2008; accepted 2 March 2009; published online 14 April 2009)

\begin{abstract}
Direct numerical simulation has been used to examine the near-field dynamics of annular gas-liquid two-phase jets. Based on an Eulerian approach with mixed fluid treatment, combined with an adapted volume of fluid method and a continuum surface force model, a mathematical formulation for the flow system is presented. The swirl introduced at the jet nozzle exit is based on analytical inflow conditions. Highly accurate numerical methods have been utilized for the solution of the compressible, unsteady, Navier-Stokes equations. Two computational cases of gas-liquid two-phase jets including swirling and nonswirling cases have been performed to investigate the effects of swirl on the flow field. In both cases the flow is more vortical at the downstream locations. The swirling motion enhances both the flow instability resulting in a larger liquid spatial dispersion and the mixing resulting in a more homogeneous flow field with more evenly distributed vorticity at the downstream locations. In the annular nonswirling case, a geometrical recirculation zone adjacent to the jet nozzle exit was observed. It was identified that the swirling motion is responsible for the development of a central recirculation zone, and the geometrical recirculation zone can be overwhelmed by the central recirculation zone leading to the presence of the central recirculation region only in the swirling gas-liquid case. Results from a swirling gas jet simulation were also included to examine the effect of the liquid sheet on the flow physics. The swirling gas jet developed a central recirculation region, but it did not develop a precessing vortex core as the swirling gas-liquid two-phase jet. The results indicate that a precessing vortex core can exist at relatively low swirl numbers in the gas-liquid two-phase flow. It was established that the liquid greatly affects the precession and the swirl number alone is an insufficient criterion for the development of a precessing vortex core. (C) 2009 American Institute of Physics. [DOI: 10.1063/1.3112740]
\end{abstract}

\section{INTRODUCTION}

A liquid spray process is a two-phase flow system with the gas, usually air, as the continuous phase and a liquid as the dispersed phase in the form of droplets or ligaments. The interactions between the two phases can occur in different ways, at different times, involving various fluid dynamic factors. In air-blasted atomizers the jet usually has an annular configuration which is characterized by the presence of two concentric shear layers near the jet nozzle exit, in comparison to one such shear layer in round jets. Many of the existing studies on annular liquid jets (e.g., Choi and Lee, ${ }^{1}$ Ibrahim and McKinney, ${ }^{2}$ and Lasheras et al. ${ }^{3}$ ) were focused on experimental visualizations and simplified mathematical models, which are very often difficult or insufficient to reveal the complex details of liquid breakup and atomization in a two-phase environment. Furthermore, the addition of swirl in annular gas-liquid two-phase jet flows is very important to atomization and spray processes, but it has not been extensively examined especially by using advanced simulation techniques.

Swirling jet flows are of practical significance since they are widely used in many industrial applications, such as in

\footnotetext{
a) Author to whom correspondence should be addressed. Telephone: $(+44)$ 1895 266685. Fax: $(+44) 1895$ 256392. Electronic mail: xi.jiang@brunel.ac.uk.
}

cyclones, in propulsion systems, in heat exchangers and most importantly in combustion and mixing. Swirling motion is regarded as an effective way to stabilize the flame near the burner exit. ${ }^{4}$ In addition, the introduction of swirling motion into a jet can lead to a higher entrainment of the ambient fluid and can enhance the flow mixing, particularly in the shear layer region. ${ }^{5,6}$ In atomization and fuel injection systems, the addition of swirl can speed up the disintegration process of the liquid sheet and alter significantly the spray characteristics. ${ }^{7}$ An understanding of the factors influencing the liquid disintegration and breakup will benefit atomizer design and aid toward a full explanation of the complex process of atomization which still remains unclear. The existing limited literature on annular liquid jets was based on experimental observations ${ }^{8-11}$ and on simple numerical formulations. $^{2,7,12}$ It is difficult to fully understand the liquid breakup mechanisms using theoretical and/or experimental approaches because of the complex interaction between the two phases.

Numerical studies of gas-liquid two-phase flows based on the traditional Reynolds-averaged Navier-Stokes (RANS) modeling approach could lead to poor predictions of highly unsteady and complex flow phenomena involving vortical structures due to the intrinsic time or ensemble averaging of the governing equations. The more advanced large-eddy simulation (LES) can be used to overcome the problems as- 
sociated with the RANS approach but it may not be sufficient to understand the detailed mechanisms in a high-speed multiphase flow, as small scales need to be modeled as well. Also, the addition of swirl cannot be easily modeled using the RANS approach due to the effects of mean flow streamline curvature. ${ }^{13}$ LES can overcome this problem associated with the RANS approach but only the major part of the turbulent motion can be resolved. ${ }^{14,15}$ In this context, direct numerical simulation (DNS) can be a very powerful tool that not only leads to a better understanding of the fluid mechanics involved, but also provides useful databases for the potential development of physical models for liquid breakup and atomization in gaseous environments. DNS was utilized by Ruith and Meiburgh ${ }^{16}$ and Kollmann et al. ${ }^{17}$ to simulate vortex breakdown in single-phase swirling jets but there is no work reported in the literature concerning DNS of annular gas-liquid two-phase swirling jet flow. Although DNS is very powerful the excessive computational cost needed to perform such complex two-phase computations is always a drawback and therefore, for the time being, such simulations have to be restricted to relatively small flow regions such as those near the jet nozzle exit.

Pierce and Moin ${ }^{18}$ were among the first to perform LES of swirling pipe flow with a sudden expansion. They used a finite volume method and obtained results for the mean velocity and the mixture fraction of a passive scalar. The influence of the level of swirl in a similar configuration was studied by Wang et al. ${ }^{19} \mathrm{Lu}$ et al. ${ }^{20}$ performed LES of a turbulent round jet issuing into a dump combustor and analyzed the interaction of the coherent structures with acoustic models of the combustor. Unsteady RANS and LES approaches were used by Wegner et al. ${ }^{21,22}$ to simulate unconfined swirling flow and spiral/helical vortical structures were obtained. DNS has been used to simulate the interface changes and turbulence in two-phase environments ${ }^{23-25}$ but the two phases were divided into two single-phase subdomains while the gas flow was considered to be incompressible. Klein ${ }^{26}$ performed DNS of a liquid sheet exhausting into a gaseous incompressible atmosphere under moderate Reynolds number. Direct computations of two-phase gas-liquid flows have been performed in axisymmetric and planar configurations $^{27-29}$ and good agreement with linear theories has been obtained. An extended study in full three dimensions is needed for detailed realization of the mixing.

This study aims at a better understanding on the flow physics of annular gas-liquid two-phase jet flows. The effects of swirl and the interactions between the two phases are examined by means of DNS. The flow characteristics are examined by direct solution of the time-dependent, nondimensional Navier-Stokes equations using highly accurate numerical schemes. Fully three-dimensional (3D) parallel simulations have been performed. In Secs. II-IV, governing equations and numerical methods used are presented followed by discussions on the results and the conclusions drawn.

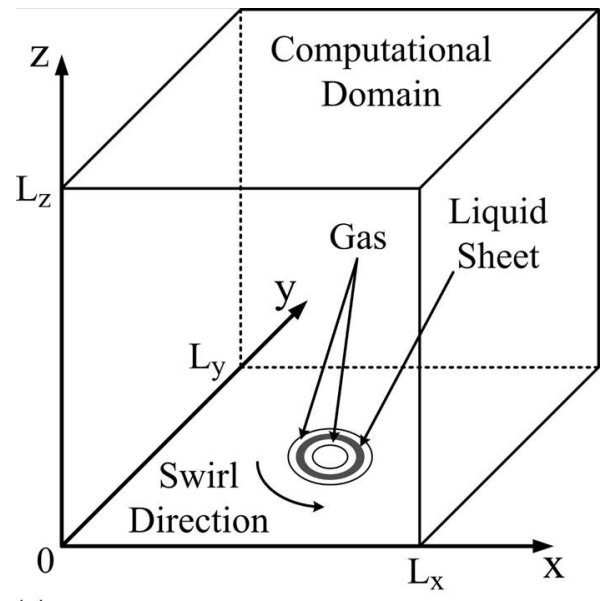

(a)

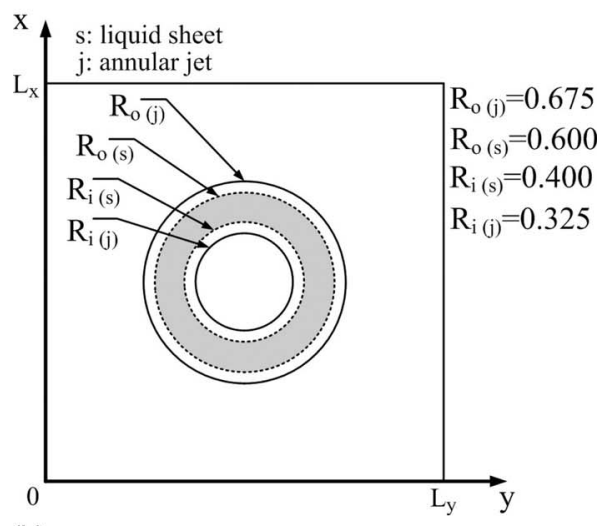

(b)

FIG. 1. Schematic of the computational domain and the inlet section. (a) Computational domain; (b) inlet section.

\section{GOVERNING EQUATIONS}

The physical problem investigated is an annular gasliquid two-phase jet issuing into an ambient environment. The flow field concerned is the region above the nozzle plane. Figure 1 shows the jet configuration and the inlet section. The flow field is described by the nondimensional timedependent Navier-Stokes equations in the Cartesian coordinate system $(x, y, z)$, where the $z$-axis is aligned with the streamwise direction of the jet while the $x-y$ plane is the cross-streamwise direction. The annular jet nozzle is located at $R_{i} \leq r \leq R_{o}$, with $r=\sqrt{\left(x-x_{0}\right)^{2}+\left(y-y_{0}\right)^{2}}$ representing the radial distance, $R_{i}$ and $R_{o}$ are the inner and outer radii of the annular jet, respectively. Using the analytical velocity profiles given in Sec. III B, the circular configuration can be represented by the square mesh associated with the Cartesian coordinates employed. The Cartesian coordinates employed avoid the singularity problems with the $1 / r$ terms at $r=0$ in the governing equations arising from the use of a cylindrical coordinate system. Reference quantities used in the normalization are the maximum streamwise velocity at the jet nozzle exit (computational domain inlet), the diameter of the annular jet $\left(R_{i}+R_{o}\right.$ measured from the middle of the annular sheet to the geometrical center of the jet nozzle exit), the ambient temperature, gas density and viscosity, and the liquid surface tension (assumed to be constant, resulting in a 
nondimensional value of one). The nondimensional quantities in the governing equations are as follows: $u, v, w$ : velocity components in the $x, y, z$ directions, respectively; $t$ is time, $\gamma$ is the ratio of specific heats of the compressible gas, $\rho$ is the gas-liquid mixture density, $\rho_{g}$ is the gas density, $\rho_{l}$ is the liquid density (assumed constant), $\mu$ is the gas-liquid mixture viscosity, $\mu_{g}$ is the gas viscosity, $\mu_{l}$ is the liquid viscosity (assumed constant), $p$ is the gas pressure, $T$ is the temperature, $Y$ is the liquid mass fraction, $\Phi$ is the liquid volume fraction, $\kappa$ is the curvature, $\sigma$ is the surface tension, $E_{T}=\rho_{g}\left[e+\left(u^{2}+v^{2}+w^{2}\right) / 2\right]$ is the total energy of the gas with $e$ representing the internal energy per unit mass, Ma is the Mach number, Pr is the Prandtl number, Re is the Reynolds number, Sc is the Schmidt number, and We is the Weber number.

The governing equations are formulated upon the conservation laws for mass, momentum, and energy and they describe both phases in a single set of equations. In the Eulerian approach with mixed-fluid treatment adopted, ${ }^{30}$ the two phases are assumed to be in local kinetic and thermal equilibrium, i.e., the relative velocities and temperatures are not significant, while the density and viscosity are considered as gas-liquid mixture properties. The liquid is assumed to be a passive scalar that is transported by the gas phase. This formulation applies only to situations where the liquid represents a dilute phase such as liquid sheets in an annular configuration investigated in this work. In the current formulation only a nonreacting isothermal flow is considered, where the two phases exchange momentum only without phase change and energy transfer taking place. Thus $u_{g}=u_{l}=u, v_{g}=v_{l}=v, w_{g}=w_{l}=w$, and $T_{g}=T_{l}=T$. The nondimensional conservation laws can be written in a vector form as

$$
\frac{\partial \boldsymbol{U}}{\partial t}+\frac{\partial \boldsymbol{E}}{\partial x}+\frac{\partial \boldsymbol{F}}{\partial y}+\frac{\partial \boldsymbol{G}}{\partial z}+\boldsymbol{H}=0
$$

where the vectors $\boldsymbol{U}, \boldsymbol{E}, \boldsymbol{F}, \boldsymbol{G}$, and $\boldsymbol{H}$ are defined as

$$
\begin{aligned}
& \boldsymbol{U}=\left(\begin{array}{c}
\rho_{g} \\
\rho u \\
\rho v \\
\rho w \\
E_{T} \\
\rho Y
\end{array}\right), \quad \boldsymbol{E}=\left[\begin{array}{c}
\rho_{g} u \\
\rho u^{2}+p-\tau_{x x} \\
\rho u v-\tau_{x y} \\
\rho u w-\tau_{x z} \\
\left(E_{T}+p\right) u+q_{x}-u \tau_{x x, g}-v \tau_{x y, g}-w \tau_{x z, g} \\
\rho u Y-\frac{1}{\operatorname{ReSc}}\left(\mu \frac{\partial Y}{\partial x}\right)
\end{array}\right], \quad \boldsymbol{F}=\left[\begin{array}{c}
\rho_{g} v \\
\rho u v-\tau_{x y} \\
{ }^{2}+p-\tau_{y y} \\
\rho u w-\tau_{y z} \\
\left(E_{T}+p\right) v+q_{y}-u \tau_{x y, g}-v \tau_{y y, g}-w \tau_{y z, g} \\
1 \\
\rho v Y-\frac{\partial Y}{\operatorname{Re~Sc}}\left(\mu \frac{\partial y}{\partial y}\right)
\end{array}\right], \\
& \boldsymbol{G}=\left[\begin{array}{c}
\rho_{g} w \\
\rho u w-\tau_{x z} \\
\rho v w-\tau_{y z} \\
\rho w^{2}+p-\tau_{z z} \\
\left(E_{T}+p\right) w+q_{z}-u \tau_{x z, g}-v \tau_{y z, g}-w \tau_{z z, g} \\
\rho w Y-\frac{1}{\operatorname{ReSc}}\left(\mu \frac{\partial Y}{\partial z}\right)
\end{array}\right], \quad \boldsymbol{H}=\left(\begin{array}{c}
0 \\
-\frac{\sigma \kappa}{\mathrm{We}} \frac{\partial \Phi}{\partial x} \\
-\frac{\sigma \kappa}{\mathrm{We}} \frac{\partial \Phi}{\partial y} \\
-\frac{\sigma \kappa}{\mathrm{We}} \frac{\partial \Phi}{\partial z} \\
0 \\
0
\end{array}\right)
\end{aligned}
$$

The constitutive relations for viscous stress components of the gas-liquid mixture are as follows:

$$
\begin{gathered}
\tau_{x x}=-\frac{2}{3} \frac{\mu}{\operatorname{Re}}\left(-2 \frac{\partial u}{\partial x}+\frac{\partial v}{\partial y}+\frac{\partial w}{\partial z}\right), \quad \tau_{x y}=\frac{\mu}{\operatorname{Re}}\left(\frac{\partial v}{\partial x}+\frac{\partial u}{\partial y}\right), \\
\tau_{y y}=-\frac{2}{3} \frac{\mu}{\operatorname{Re}}\left(\frac{\partial u}{\partial x}-2 \frac{\partial v}{\partial y}+\frac{\partial w}{\partial z}\right), \quad \tau_{x z}=\frac{\mu}{\operatorname{Re}}\left(\frac{\partial w}{\partial x}+\frac{\partial u}{\partial z}\right),
\end{gathered}
$$

$\tau_{z z}=-\frac{2}{3} \frac{\mu}{\operatorname{Re}}\left(\frac{\partial u}{\partial x}+\frac{\partial v}{\partial y}-2 \frac{\partial w}{\partial z}\right), \quad \tau_{y z}=\frac{\mu}{\operatorname{Re}}\left(\frac{\partial w}{\partial y}+\frac{\partial v}{\partial z}\right)$

while the gas phase viscous stress and heat flux components are expressed as

$$
\begin{aligned}
\tau_{x x, g} & =-\frac{2}{3} \frac{\mu_{g}}{\operatorname{Re}}\left(-2 \frac{\partial u}{\partial x}+\frac{\partial v}{\partial y}+\frac{\partial w}{\partial z}\right), \\
\tau_{x y, g} & =\frac{\mu_{g}}{\operatorname{Re}}\left(\frac{\partial v}{\partial x}+\frac{\partial u}{\partial y}\right),
\end{aligned}
$$




$$
\begin{aligned}
& \tau_{y y, g}=-\frac{2}{3} \frac{\mu_{g}}{\operatorname{Re}}\left(\frac{\partial u}{\partial x}-2 \frac{\partial v}{\partial y}+\frac{\partial w}{\partial z}\right), \\
& \tau_{x z, g}=\frac{\mu_{g}}{\operatorname{Re}}\left(\frac{\partial w}{\partial x}+\frac{\partial u}{\partial z}\right), \\
& \tau_{z z, g}=-\frac{2}{3} \frac{\mu_{g}}{\operatorname{Re}}\left(\frac{\partial u}{\partial x}+\frac{\partial v}{\partial y}-2 \frac{\partial w}{\partial z}\right), \\
& \tau_{y z, g}=\frac{\mu_{g}}{\operatorname{Re}}\left(\frac{\partial w}{\partial y}+\frac{\partial v}{\partial z}\right), \\
& q_{x}=\frac{-\mu_{g}}{(\gamma-1) \mathrm{Ma}^{2} \operatorname{Pr} \operatorname{Re}} \frac{\partial T}{\partial x}, \\
& q_{y}=\frac{-\mu_{g}}{(\gamma-1) \mathrm{Ma}^{2} \operatorname{Pr} \operatorname{Re}} \frac{\partial T}{\partial y}, \\
& (\gamma-1) \operatorname{Ma}^{2} \operatorname{Pr} \operatorname{Re} \frac{\partial T}{\partial z} .
\end{aligned}
$$

Assuming the gas medium as an ideal gas, the governing equations for the gas-liquid two-phase flow system include also the perfect gas law, given by

$$
p=\frac{\rho_{g} T}{\gamma \mathrm{Ma}^{2}} .
$$

The physics of the gas-liquid interface are computed and analyzed using the volume of fluid (VOF) method by Hirt and Nichols, ${ }^{31}$ which employs the liquid volume fraction. The liquid volume fraction works as an indicator to identify the different fluids. A liquid volume fraction value of one, $\Phi=1$, corresponds to pure liquid and a value of zero, $\Phi=0$, corresponds to pure gas. In between the two values, $0<\Phi<1$, a gas-liquid interface region exists and the fluid is considered as a mixture. In this study, the original VOF method has been adapted to solve an equation for the liquid mass fraction $Y$ rather than the volume fraction $\Phi$ in order to suit the compressible gas phase formulation. ${ }^{27-29}$ From their definitions, a relation between liquid volume fraction and liquid mass fraction can be derived as

$$
\Phi=\frac{\rho_{g} Y}{\rho_{l}-\left(\rho_{l}-\rho_{g}\right) Y} .
$$

Following Gueyffier et al., ${ }^{32}$ the density and viscosity of the gas-liquid two-phase fluid flow are considered as functions of the liquid volume fraction and densities and viscosities of both phases, given by

$$
\begin{aligned}
& \rho=\Phi \rho_{l}+(1-\Phi) \rho_{g}, \\
& \mu=\Phi \mu_{l}+(1-\Phi) \mu_{g} .
\end{aligned}
$$

Equations (7) and (8) are utilized in conjunction with the VOF method, to account for the contributions of the two individual phases to the mixture properties.

The gas-liquid interface dynamics are resolved using a continuum surface force (CSF) model developed by Brack- bill et al., ${ }^{33}$ which represents the surface tension effect as continuous volumetric force acting within the region where the two phases coexist. The CSF model overcomes the problem of directly computing the surface tension integral that appears in the Navier-Stokes momentum equations, which requires the exact shape and location of the interface. In the CSF model, the surface tension force in its nondimensional form, as it appears in Eq. (2), can be approximated as $\sigma \kappa /$ We $\nabla \Phi$, with the curvature of the interface given by

$$
\kappa=-\nabla \cdot\left(\frac{\nabla \Phi}{|\nabla \Phi|}\right) \text {. }
$$

\section{NUMERICAL SOLUTION PROCEDURES}

\section{A. Time-advancement and discretization}

The numerical methods include the high-order finitedifference schemes for time advancement and spatial discretization. The governing equations are integrated forward in time using a third-order compact-storage fully explicit Runge-Kutta scheme. ${ }^{34}$ The solution variables $\left(\rho_{g}, \rho u, \rho v\right.$, $\rho w, E_{T}$, and $\left.\rho Y\right)$ in Eq. (2) are advanced in time using a three-step compact-storage third-order Runge-Kutta scheme of the family derived by Wray. Two storage locations are employed for each time-dependent variable and at each substep at these locations, say $Q_{1}$ and $Q_{2}$ with $Q$ representing the solution variables, are updated simultaneously as follows:

$$
Q_{1}^{\text {new }}=a_{1} Q_{1}^{\text {old }} \Delta t+Q_{2}^{\text {old }}, \quad Q_{2}^{\text {new }}=a_{2} Q_{1}^{\text {old }} \Delta t+Q_{2}^{\text {old }} .
$$

The constants $\left(a_{1}, a_{2}\right)$ in Eq. (10) are chosen to be $(2 / 3,1 / 4)$ for substep $1,(5 / 12,3 / 20)$ for substep 2 , and $(3 / 5,3 / 5)$ for substep 3. At the beginning of each full time step, $Q_{1}$ and $Q_{2}$ are equal. The data in $Q_{1}$ are used to compute $\partial \boldsymbol{U} / \partial t$ in Eq. (1). The computed $\partial \boldsymbol{U} / \partial t$ is stored in $Q_{1}$ to save storage (overwriting the old $Q_{1}$ ). Equation (10) is then used to update $Q_{1}$ and $Q_{2}$. In Eq. (10), $\Delta t$ is the time step, which is limited by the Courant-Friedrichs-Lewy condition for stability.

During the time advancement, the density and viscosity of the gas-liquid two-phase flow system are calculated according to Eqs. (7) and (8), using the volume fraction $\Phi$ calculated from Eq. (6). However, the liquid mass fraction $Y$ in Eq. (6) needs to be calculated from the solution variable $\rho Y$ first. Using $q$ to represent $\rho Y$ at each time step, the liquid mass fraction $Y$ can be calculated as

$$
Y=\frac{\rho_{l} q}{\rho_{l} \rho_{g}+\left(\rho_{l}-\rho_{g}\right) q} .
$$

Equation (11) can be derived from Eqs. (6) and (7). At each time step, Eq. (11) is used first to calculate the liquid mass fraction, Eq. (6) is then used to calculate the liquid volume fraction and Eqs. (7) and (8) are finally used to update the mixture density and viscosity.

Spatial differentiation is achieved using the sixth-order compact (Padé) finite-difference scheme of Lele, ${ }^{35}$ which was extensively used in DNS of fluid flow problems. Solutions for the discretized equations are obtained by solving the 
tridiagonal system of equations using a simplified form of Gaussian elimination.

\section{B. Boundary conditions}

The 3D computational domain is bounded by the inflow and the outflow boundaries in the streamwise direction and open boundaries with the ambient field in the jet radial (cross-streamwise) direction. The nonreflecting characteristic boundary conditions due to Thompson ${ }^{36}$ are applied at the open boundaries, which prevent the wave reflections from the outside of the computational domain. The nonreflecting boundary conditions are also used at the outflow boundary in the streamwise direction. The spurious wave reflections from outside the boundary have been controlled by using a sponge layer $\left(L_{z s}<z \leq L_{z}\right)$ next to the outflow boundary. ${ }^{37}$ The strategy of using a sponge layer is similar to that of "sponge region" or "exit zone" (Mitchell et al. ${ }^{38}$ ), which has been proved to be very effective in controlling the wave reflections through the outflow boundary. The results of the sponge layer are unphysical and therefore are not used in the data analysis.

The inflow conditions at the jet nozzle exit need careful attention. They represent the initial mass and momentum distributions of the annular gas-liquid two-phase jet. Under swirling conditions, they must be able to represent the amount of swirl at the jet nozzle exit as realistically as possible. Based on the concept of Pierce and Moin ${ }^{39}$ for numerical generation of equilibrium swirling inflow conditions, analytical solutions of the axial and azimuthal velocity components were derived, which enable simple and precise definition of the desired swirl level. ${ }^{40}$ The analytical profiles of axial and azimuthal velocities are given as

$$
\begin{aligned}
& w=-\frac{1}{4} \frac{f_{x}}{\mu}\left(r^{2}-\frac{R_{i}^{2}-R_{o}^{2}}{\ln R_{i}-\ln R_{o}} \ln r+\frac{R_{i}^{2} \ln R_{o}-R_{o}^{2} \ln R_{i}}{\ln R_{i}-\ln R_{o}}\right), \\
& u_{\theta}=-\frac{1}{3} \frac{f_{\theta}}{\mu}\left(r^{2}-\frac{R_{i}^{2}+R_{i} R_{o}+R_{o}^{2}}{R_{i}+R_{o}} r+\frac{R_{i}^{2} R_{o}^{2}}{R_{i}+R_{o}} \frac{1}{r}\right) .
\end{aligned}
$$

Equation (12) only holds for the jet annulus $R_{i} \leq r \leq R_{o}$, and zero velocities have been specified outside the jet annulus. In Eq. (12) $f_{x}$ and $f_{\theta}$ can be defined by the maximum velocities at the inflow boundary. For a unit maximum velocity, which is often the case when a nondimensional form of the governing equations is employed, the constant $f_{x}$ is defined as

$$
f_{x}=-\frac{8 \mu\left(\ln R_{o}-\ln R_{i}\right)}{R_{o}^{2}-R_{i}^{2}+R_{i}^{2} \ln \left[\frac{R_{i}^{2}-R_{o}^{2}}{2\left(\ln R_{i}-\ln R_{o}\right)}\right]-R_{o}^{2} \ln \left[\frac{R_{i}^{2}-R_{o}^{2}}{2\left(\ln R_{i}-\ln R_{o}\right)}\right]-2 R_{i}^{2} \ln R_{o}+2 R_{o}^{2} \ln R_{i}} .
$$

The parameter $f_{\theta}$ defines the degree of swirl. For known $w$ and $u_{\theta}$ the swirl number can be calculated from

$$
S=\frac{\int_{R_{i}}^{R_{o}} w u_{\theta} r^{2} d r}{R_{o} \int_{R_{i}}^{R_{o} w^{2} r d r}}
$$

A certain swirl number can be conveniently achieved by adjusting the constant $f_{\theta}$ in Eq. (12). From the azimuthal velocity $u_{\theta}$, the cross-streamwise velocity components at the inflow can be specified by $u=-u_{\theta} y / r$ and $v=u_{\theta} x / r$. At the inflow boundary, the liquid mass fraction profile has been specified using a distribution similar to the streamwise (axial) velocity profile.

The mean velocity at the inflow was perturbed by a flapping mode which contains two helical modes with the same frequency and amplitude. ${ }^{41}$ The velocity components at the jet nozzle exit $z=0$ can be given as

$$
\begin{aligned}
& u=\bar{u}+A \sin \left(m \varphi-2 \pi f_{0} t\right), \quad v=\bar{v}+A \sin \left(m \varphi-2 \pi f_{0} t\right), \\
& w=\bar{w}+A \sin \left(m \varphi-2 \pi f_{0} t\right),
\end{aligned}
$$

where $A$ is the amplitude of disturbance, $m$ is the mode number, $\varphi$ is the azimuthal angle, and $f_{0}$ is the excitation frequency. The amplitude of the disturbance is $1 \%$ of the maximum value of the streamwise velocity. The nondimensional frequency (Strouhal number) of the unsteady disturbance is chosen to be $f_{0}=0.3$, which is the most unstable mode leading to the jet preferred mode of instability. ${ }^{42}$ Two helical disturbances of $m=1$ and $m=-1$ were superimposed on the temporal disturbance. ${ }^{41}$

\section{COMPUTATIONAL DETAILS}

Three computational cases have been performed in order to investigate the effects of swirl and the interactions between the two phases on the flow development of an annular gas-liquid two-phase jet, including nonswirling and swirling gas-liquid two-phase jets, and a swirling gas jet to be compared to the corresponding two-phase jet. The width of the annular sheet is 0.35 while the thickness of the liquid sheet is 0.2 and it is located in the middle of the annulus, as shown in Fig. 1. The input parameters correspond to diesel injection into compressed air at approximately $15 \mathrm{MPa}$ and $300 \mathrm{~K}$, where the liquid surface tension is about $0.025 \mathrm{~N} / \mathrm{m}$. Using the reference quantities defined in Sec. II, the input parameters used in the simulations are $^{43}$ Mach number $\mathrm{Ma}=0.4$, Reynolds number $\operatorname{Re}=2000$, Prandtl number $\operatorname{Pr}=0.76$, Schmidt number $\mathrm{Sc}=0.76$, Weber number $\mathrm{We}=240$, and ratio of specific heats $\gamma=1.64$. For the swirling cases the swirl number is taken to be $S=0.4$, with the nonswirling case having a zero-swirl number. 
The dimensions of the computational box used are $L_{x}=L_{y}=L_{z}=10$. The grid system is of $512 \times 512 \times 512$ nodes with a uniform distribution in each direction. The grid determines the scales that are resolved. The Kolmogorov length scale is commonly quoted as the smallest scale that needs to be resolved in DNS-type simulations ${ }^{44}$ and may be defined as ${ }^{45} \eta_{K}=L_{\mathrm{ref}}^{*} / \operatorname{Re}_{t}^{3 / 4}$, where $L_{\mathrm{ref}}^{*}$ is the dimensional reference length scale and $\mathrm{Re}_{t}$ is the turbulent Reynolds number. The $L_{\text {ref }}^{*}$ corresponding to the nominal Reynolds and Mach numbers used in the simulations is around $2 \mu \mathrm{m}$. Based on the nondimensional input parameters and the velocity fluctuations observed in the flow fields (such as those shown in Fig. 9), the Kolmogorov length scale $\eta_{K}$ can be roughly estimated to be around $0.3 \mu \mathrm{m}$, which is larger than the grid spacing $\eta_{\text {grid }} \approx 0.04 \mu \mathrm{m}$. However, the $\eta_{K}$ estimated here might not be a good criterion to assess the quality of the simulation because of the transitional rather than fully turbulent nature of the flow. In this study, grid and time step dependence tests were also performed by doubling the grid points in one direction (doubling the grid points in all directions proved to be too costly to perform) and halving the time step, which did not show appreciable changes in the results. The results presented are therefore considered to be of adequate resolution. Obviously, the physical scales of the problem corresponding to the nondimensional parameters used are very small. However, tests showed that changing the Mach number from 0.4 to 0.05 did not lead to appreciable changes in the solution, indicating that the DNS results may be applicable for physical problems that are ten times larger than that indicated by the $L_{\text {ref }}^{*}$. The scaled-up physical scales correspond to those of microdiesel injector nozzles ${ }^{46}$ and microelectromechanical system-type nozzles. ${ }^{47}$ Although scaling to larger configurations can be approximate or even distorted, DNS results of this type can be used to gain better insights into practical problems. Under this perspective the results are considered to be useful for understanding fuel injection processes in practical applications.

Parallel computations have been performed, under the message passing interface environment, on an IBM pSeries 690 Turbo Supercomputer utilizing 512 processors. The 3D parallel DNS code used was developed from the 3D parallel DNS code for gas jets ${ }^{48,49}$ based on the gas-liquid two-phase flow formulation used in the axisymmetric and planar simulations. ${ }^{27-29}$ The excessive computational cost needed to perform the complex two-phase DNS (around 300000 allocation units on HPCx per simulation) limits the computation to regions close to the jet nozzle exit. The results are discussed in terms of the instantaneous and time- and spatialaveraged flow properties. For a consistent comparison, the number of contours has been kept the same in all computational cases for the contour plots shown.

\section{RESULTS AND DISCUSSION}

\section{A. Instantaneous flow characteristics}

Figure 2 shows the instantaneous isosurfaces of enstrophy $\Omega=\left(\omega_{x}^{2}+\omega_{y}^{2}+\omega_{z}^{2}\right) / 2$ and liquid volume fraction $\Phi$ at the nondimensional time of $t=30.0$. The individual vorticity components are defined as $\omega_{x}=\partial w / \partial y-\partial v / \partial z, \omega_{y}=\partial u / \partial z$
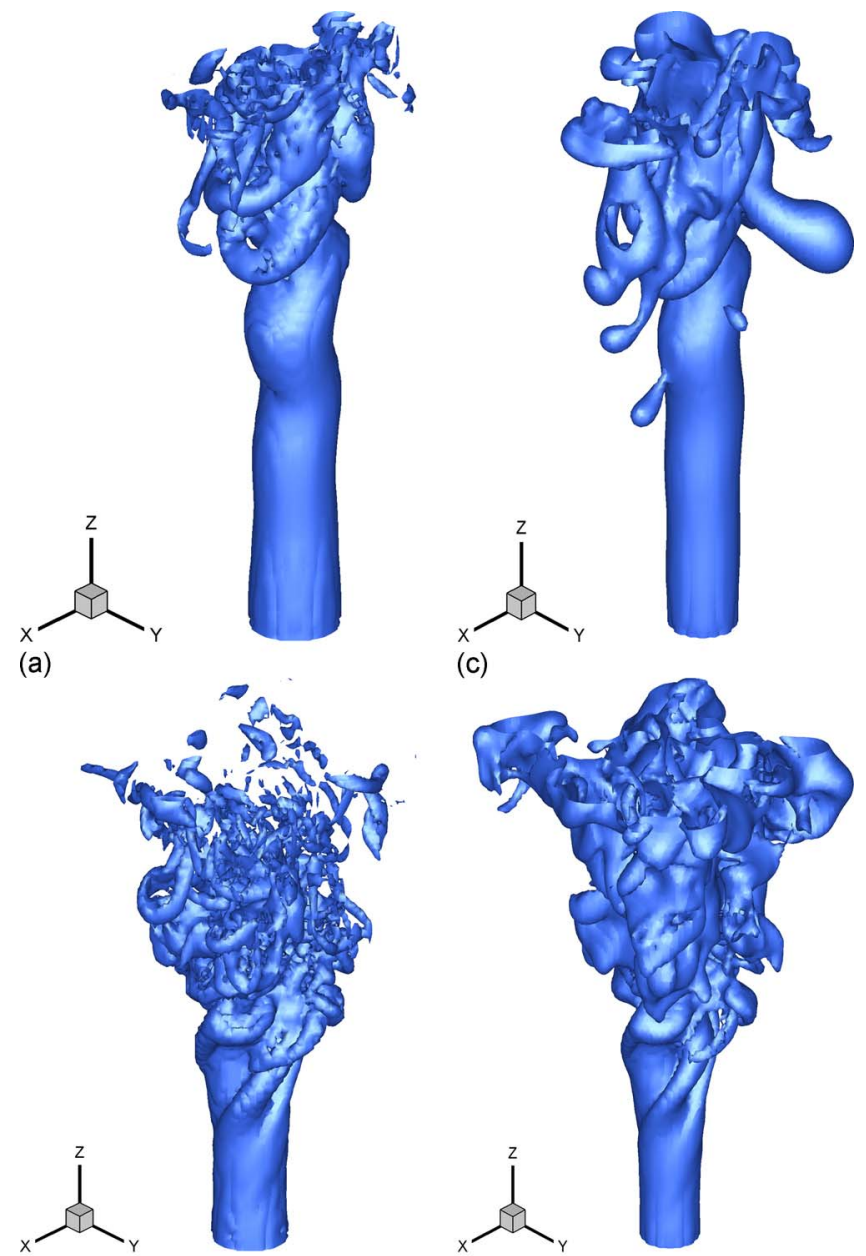

(c)

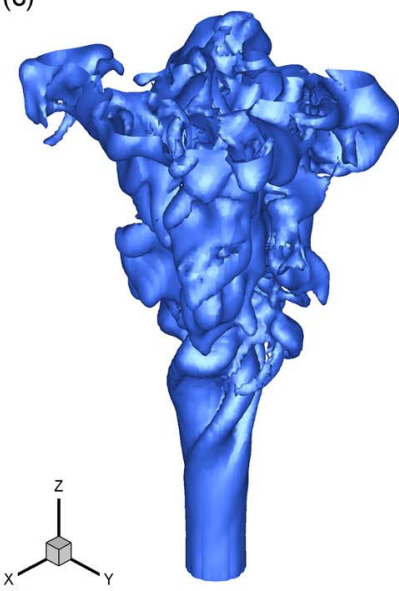

(d)

FIG. 2. (Color online) Instantaneous isosurfaces of enstrophy and liquid volume fraction at $t=30.0$. (a) Enstrophy $(S=0)$; (b) enstrophy $(S=0.4)$; (c) liquid volume fraction $(S=0)$; (d) liquid volume fraction $(S=0.4)$.

$-\partial w / \partial x$, and $\omega_{z}=\partial v / \partial x-\partial u / \partial y$. From Fig. 2, it is evident that the dispersion of the liquid is dominated by large-scale vortical structures formed at the jet primary stream due to the Kelvin-Helmholtz-type shear layer instability. The presence of streamwise vorticity, which is absent in idealized axisymmetric and planar configurations, ${ }^{27-29}$ is generated by $3 \mathrm{D}$ vortex stretching and interaction. The flow vorticity dominates the dispersion of the liquid as indicated by the similar structures of the enstrophy and liquid volume fraction. For the nonswirling case, it is interesting to notice that there is no formation of significant vortical structures between the jet nozzle exit at $z=0$ and the location of $z=5.0$. After $z=5.0$, the Kelvin-Helmholtz instability grows rapidly leading to the formation of elongated finger-type vortices. For the swirling case, an anticlockwise rotating motion was introduced into the annular gas-liquid two-phase jet at the nozzle exit. ${ }^{40}$ This rotating pattern is shown in Fig. 1. The introduction of swirling motion results in faster initiation of the Kelvin-Helmholtz instability compared to the nonswirling case. This is shown in the formation of vortical structures at relatively upstream locations in Fig. 2(b). The vortical structures show similar finger-type shapes as in the nonswirling case and they tend to collapse at further downstream loca- 

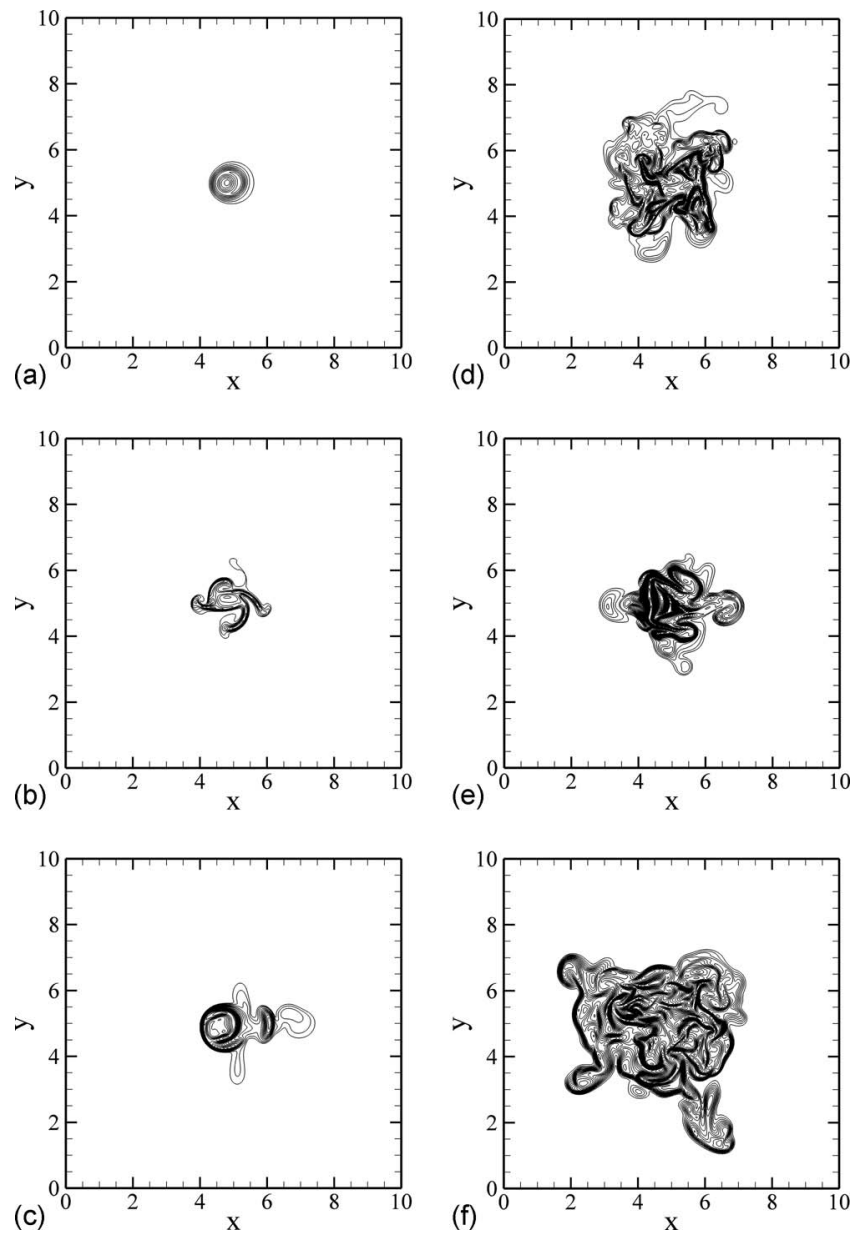

FIG. 3. Instantaneous liquid volume fraction contours in various streamwise planes at $t=30.0$. (a) $z=4.0(S=0)$; (b) $z=4.0(S=0.4)$; (c) $z=6.0(S=0)$; (d) $z=6.0(S=0.4)$; (e) $z=8.0(S=0)$; (f) $z=8.0(S=0.4)$.

tions as the flow progresses. By comparing Figs. 2(a) and 2(b) it is clear that the swirling case is more vortical. As a result, the cross-streamwise liquid dispersion is enhanced by the addition of swirl. Swirl extends the curved shear layer and promotes mixing; therefore, the liquid spreads more in the cross-streamwise direction and forms a conical shape from the inlet to further downstream locations.

To further elucidate the liquid distribution, Fig. 3 shows the instantaneous liquid volume fraction contours in various streamwise planes for both gas-liquid two-phase cases. The nonswirling case shows a relatively undistorted liquid distribution at $z=4.0$. At $z=6.0$ the liquid undergoes disintegration from its original circular distribution while it further expands downstream at $z=8.0$. In the meantime, the liquid distributions for the swirling case are quite different. The anticlockwise swirling motion has a direct impact on the liquid distribution. This is evident in Fig. 3(b) where the liquid starts to develop extended branches in both directions, compared to the nonswirling case, where no significant liquid distribution is noticed at $z=4.0$. Further downstream, the crossstreamwise liquid distribution is larger and expands more in both $x$ - and $y$-directions compared to the zero-swirl case. For the swirling case, the increase in liquid dispersion is due to the presence of swirl which gives rise to centrifugal forces,
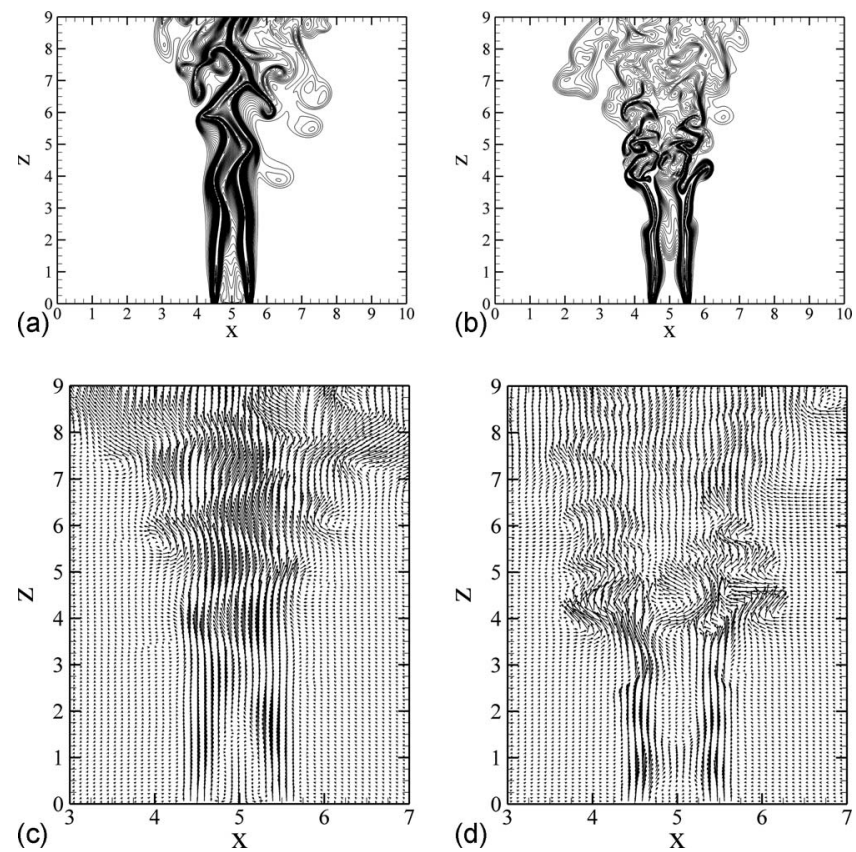

FIG. 4. Instantaneous liquid volume fraction contours and velocity vectors in $y=5.0$ plane at $t=30.0$. (a) Liquid volume fraction $(S=0)$; (b) liquid volume fraction $(S=0.4)$; (c) velocity vectors $(S=0)$; (d) velocity vectors $(S=0.4)$.

causing the liquid sheet to move outwards in the radial direction. The liquid distribution tendencies observed here were also experimentally observed by Ramamurthi and Tharakan. ${ }^{9}$

Figure 4 shows the instantaneous liquid volume fraction contours and velocity vectors in the $y=5.0$ plane at $t=30.0$. In both cases the annular liquid branches meet further downstream as the flow develops, initiating the liquid deformation. The nonswirling case undergoes significant liquid dispersion and spreading from the annular column after $z=6.0$, while the swirling case shows earlier occurrence of this phenomenon, starting at $z=5.0$. A noticeable feature in Fig. 4 is that a small amount of liquid is present adjacent to the jet nozzle exit, in the nonswirling case, lying inside and above the nozzle annulus between $x=4.75$ and $x=5.25$. This is associated with the development of a geometrical recirculation zone (GRZ) adjacent to the jet nozzle exit, which is a common feature of annular jet flows. ${ }^{5}$ The velocity reversals, revealed by a close examination of the velocities inside the jet column, tend to drag the liquid toward the inner vicinity of the annular column. On the contrary, the swirling case shows no liquid present in the region inside the jet column and immediately adjacent to the nozzle, where an empty envelope is observed from $z=0$ to $z=1.5$. The development of the recirculation zone will be further discussed later on. An important feature in Fig. 4 is that at downstream locations, between $z=7.0$ and $z=9.0$, the swirling flow field, although more vortical, is more homogeneous in the sense that vorticity is more evenly distributed. This is also evident from the velocity vector maps in Figs. 4(c) and 4(d) where the homogeneity is represented by the more uniform velocity field without obvious presence of large-scale structures at the downstream locations mentioned. The zero-swirl case devel- 

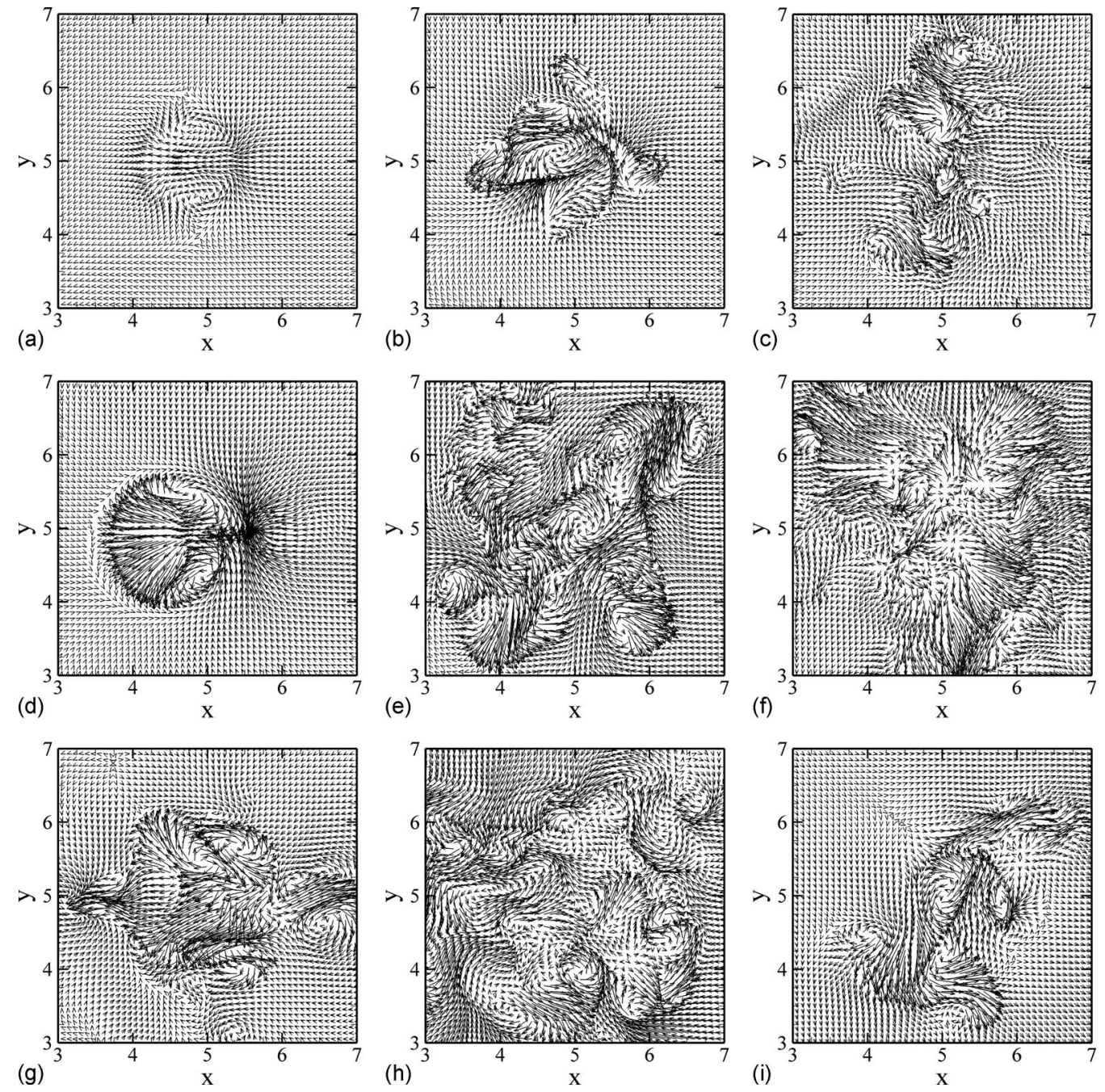

FIG. 5. Instantaneous velocity vector maps in various streamwise planes at $t=30.0$. (a) $z=4.0(S=0)$; (b) $z=4.0(S=0.4)$; (c) $z=4.0$ ( $S=0.4$, gas jet); (d) $z$ $=6.0(S=0)$; (e) $z=6.0(S=0.4)$; (f) $z=6.0$ ( $S=0.4$, gas jet); (g) $z=8.0(S=0)$; (h) $z=8.0(S=0.4)$; (i) $z=8.0$ ( $S=0.4$, gas jet).

ops dynamic vortical structures further downstream, as shown in Fig. 4(c), causing an inhomogeneous liquid distribution. The downstream homogeneity observed in Figs. 4(b) and 4(d), is a direct consequence of enhanced mixing due to the swirling mechanism.

The instantaneous velocity vector maps in various crossstreamwise planes are shown in Fig. 5. For clarity reasons the vector plots are shown only for a limited number of grid points, which is significantly less than the total number of grid points. In both cases, complex vortical structures develop at downstream locations. For the nonswirling case, complex structures can be observed at downstream locations, which are evident at further downstream location of $z=8.0$. The structure development is because of the KelvinHelmholtz instability triggered by the helical modes in the small external perturbation applied at the nozzle exit. The rather stable flow field observed at the upstream location $z=4.0$ is due to the nondevelopment of the instability at this location. For the swirling case, at $z=6.0$ and $z=8.0$, the velocity distributions become more complex with less compactness, compared to $z=4.0$, due to the downstream collapsing of the vortical structures to smaller ones. At the downstream location $z=8.0$, the swirling case is more vortical but also more homogeneous, as shown in Fig. 5(h), consistent with the observations in Fig. 4. For the swirling gas jet case, it is interesting to notice that the spreading at $z=8.0$ is less than that that of the gas-liquid two-phase jet. The absence of the liquid reduces the cross-streamwise spreading of the jet at this downstream location as shown by the comparison between Figs. 5(h) and 5(i).

A close examination of Figs. 5(b) and 5(e) revealed the existence of an inner anticlockwise rotating structure near the geometrical center of the jet, which is known as the precessing vortex core $(\mathrm{PVC}){ }^{4}$ The center of the PVC is located approximately at $(x=4.75, y=5.10)$ in Fig. 5(b). GarcíaVillalba and Fröhlich ${ }^{15}$ stated that PVC is mainly associated with strong swirls and is not expected for low swirl numbers $(S<0.55)$. This was observed for swirling round jets. ${ }^{4,50} \mathrm{In}$ annular configurations, there is experimental evidence that at low swirl numbers $(S<0.6)$, a PVC structure can exist. ${ }^{50-52}$ The PVC can be considered as having two major components: the vortex core rotating around the symmetry axis while at the same time the vortex spinning around its own 

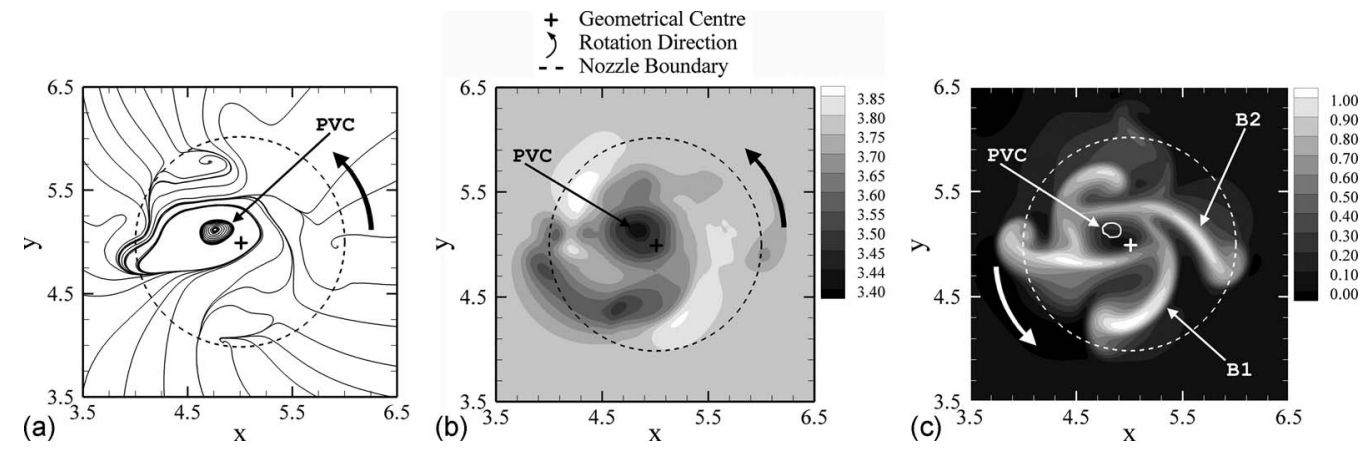

FIG. 6. Instantaneous stream traces, gas pressure, and axial velocity $(w)$ in $z=4.0$ plane at $t=30.0$ of the swirling case. (a) Stream traces; (b) gas pressure; (c) axial velocity.

axis. To better identify the PVC, Fig. 6 shows the instantaneous stream traces, contours of gas pressure, and axial velocity $(w)$ at $z=4.0$. The self-rotating pattern of the PVC is evident in Fig. 6(a). The PVC location is also indicated by the low-pressure region which is shown by an arrow in Fig. 6(b). In the same region, positive axial velocity of intermediate magnitude can be identified, as shown in Fig. 6(c). The maximum axial velocity occurs in curved regions indicated as B1 and B2 due to the swirl. The DNS results reported herein, for an annular flow with low swirl number $(S=0.4)$ are in agreement with the experimental observations of Al-Abdeli and Masri ${ }^{51,52}$ regarding the PVC development.

Figure 7 shows the instantaneous stream traces, contours of gas pressure and axial velocity $(w)$ at $z=4.0$ for the swirling gas jet case. The important feature in Fig. 7 is that no PVC is developed in this case. Although the configuration and the swirl number have been kept the same as those in the two-phase swirling jet, the single-phase gas jet does not develop a PVC as that in the two-phase flow. This is due to the fact that the swirling gas jet has lower azimuthal momentum flux compared to the swirling two-phase case, leading to weaker swirling effects in the single-phase case. The swirl number alone is not a sufficient criterion for PVC development. Other factors affecting the recirculation patterns and consequently the PVC growth include also the geometrical characteristics of the nozzle itself. ${ }^{53} \mathrm{~A}$ PVC can exist in annular swirling jet flows under certain conditions, while the PVC in a two-phase flow environment may develop at relatively low swirl numbers.
Profiles of the instantaneous streamwise velocity at the jet center line at various time instants are shown in Figs. 8(a) and $8(\mathrm{~b})$, for the nonswirling and swirling two-phase jets, respectively. In Fig. 8(a) it is clear that the velocity profiles from $z=0$ to $z=1.75$ are overlapping, and have negative values, confirming the GRZ formation adjacent to the jet nozzle exit which is due to the characteristics of the annular configuration and the presence of two concentric shear layers. Such recirculation zones were also experimentally identified by Sheen $e t a l{ }^{5}$ After $z=3.0$, large positive velocity fluctuations are present indicating the formation of unsteady/ dynamic vortical structures further downstream. For the swirling case, significant negative velocity regions are present between $z=1.0$ and $z=3.0$, showing the formation of a central recirculation zone (CRZ). The positioning of both the GRZ and the CRZ is not time dependent, although small velocity variations can be observed for the three time instants shown in Fig. 8(b) for the CRZ, a feature which is not obvious in the GRZ where the velocity profiles are almost overlapping. After $z=4.0$, velocity fluctuations are present indicating the dynamic movements of the downstream vortices. In Fig. 8, it is evident that the two cases differ significantly. Additional analysis is presented in an effort to better understand the flow physics and the changes occurring in the flow field when a swirling motion is applied at the nozzle exit, based on analyzing the velocity histories.

Figure 9 shows, by means of time traces, the streamwise velocities at the jet center line for both cases at four locations starting from $z=2.0$ and progressing downstream to $z=4.0$,
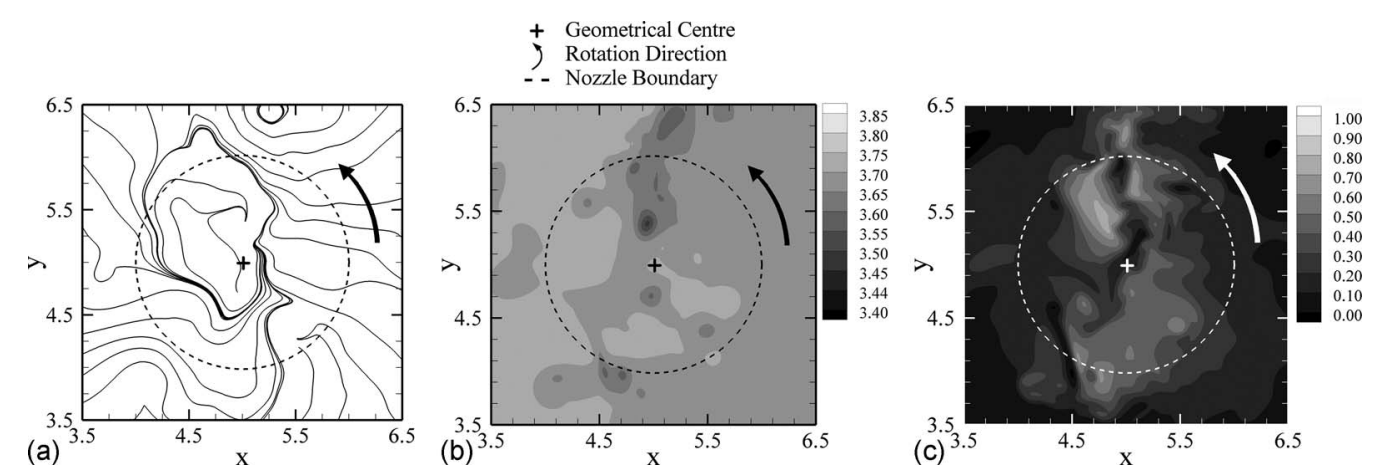

FIG. 7. Instantaneous stream traces, gas pressure, and axial velocity $(w)$ in $z=4.0$ plane at $t=30.0$ of the swirling gas jet case. (a) Stream traces; (b) gas pressure; (c) axial velocity. 

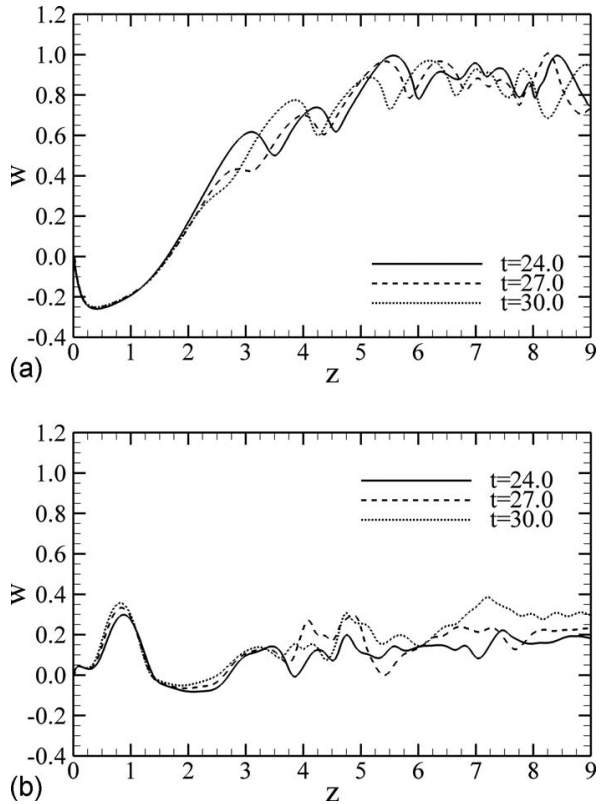

FIG. 8. Instantaneous streamwise velocity profiles at the jet center line at different time instants. (a) $S=0$; (b) $S=0.4$.

$z=6.0$, and $z=8.0$. At $z=2.0$, the velocity profile is smooth without any significant fluctuations for both cases. The important difference between the two cases at $z=2.0$ is that for the swirling case, the velocity magnitudes show negative values. This is due to the fact that this particular position lies in the heart of the CRZ. For the nonswirling case, at further downstream locations, significant velocity fluctuations are present, associated with the formation and convection of large-scale vortical structures which change the local velocity. The swirling case shows smaller variations in velocity
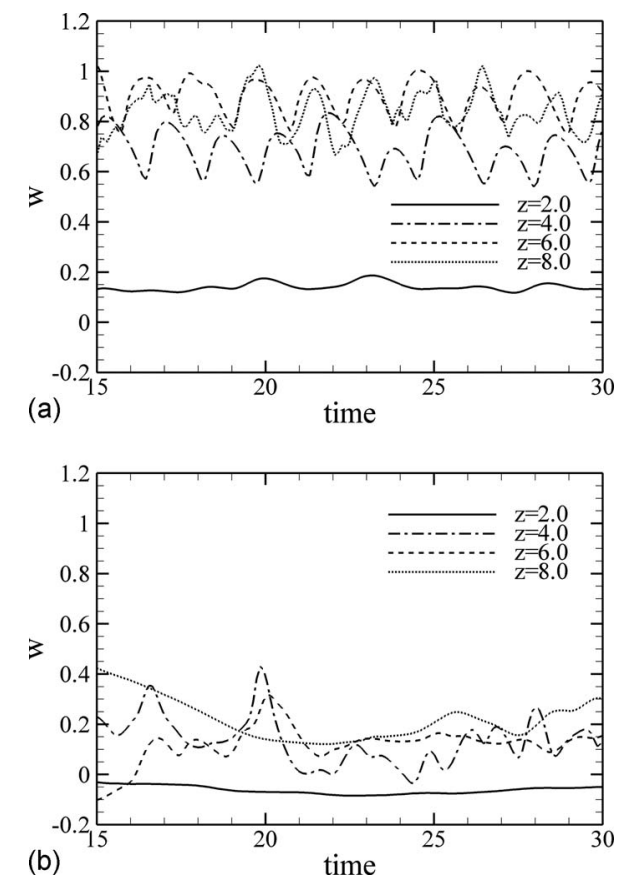

FIG. 9. Streamwise velocity histories along the jet center line. (a) $S=0$; (b) $S=0.4$.
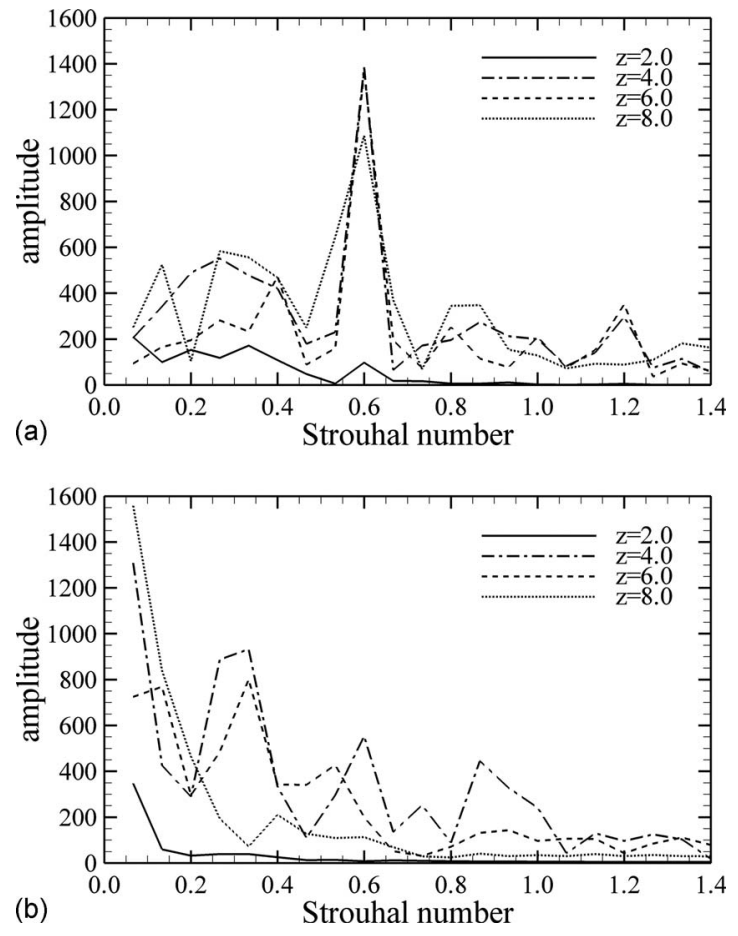

FIG. 10. Fourier energy spectra of the streamwise velocities along the jet center line. (a) $S=0$; (b) $S=0.4$.

amplitudes at $z=4.0$ and $z=6.0$ due to the swirl enhanced mixing of the flow with the ambient fluid. An important feature in Fig. 9(b) is that at $z=8.0$ no significant fluctuations are present, compared to the nonswirling case. The swirl tends to enhance the bulk mixing of the jet with the quiescent ambient environment, thus decreases the streamwise velocity magnitudes and results in a more homogeneous flow field at the downstream location $z=8.0$. An overall picture of the homogeneous vortical flow field can be seen in Fig. 5(h).

Figure 10 shows the energy spectra of the streamwise velocities at four locations in the flow fields, corresponding to Fig. 9. The energy spectra were computed from the streamwise velocity histories along the jet center line shown in Fig. 9 (where the initial transient had been discarded) using a fast Fourier transform. Figure 10(a) shows the energy spectra of the gas-liquid nonswirling case while Fig. 10(b) shows the spectral distribution in the gas-liquid swirling case. In Fig. 10(a) it is noticed that the dominant frequency has a value of $\mathrm{St}=0.6$ which is doubled compared to the small external perturbation applied at the inlet $(\mathrm{St}=0.3)$. This is due to the interaction of two flapping modes. The amplitudes of the energy spectra at the downstream locations are significantly larger than that at the upstream location of $z$ $=2.0$ due to the growth of the Kelvin-Helmholtz instability. It is interesting to notice that the swirling gas-liquid case, as shown in Fig. 10(b), shows no nonzero dominant frequencies. This is due to the swirling mechanism which affects the flow vortical structures. The swirl significantly changes the velocity distributions, and consequently the energy spectra are greatly affected. However, it is worth noting that the energy spectra can be influenced by the relatively small in- 
terval of the time history data ( $t=15-30$ as shown in Fig. 9), which may cause inaccuracy in the dominant frequencies revealed especially for the swirling case.

\section{B. Spatially and temporally averaged flow characteristics}

In this study, spatially and temporally averaged flow properties have also been calculated to examine the flow differences between the three cases performed. The time interval used for the calculation of the time-averaging properties is between $t_{1}=23.3$ and $t_{2}=30.0$, after the flow has developed. The spatial averaging is performed in the azimuthal direction so the results can also be presented in a cylindrical coordinate system. The jet center line $r=0$ corresponds to $(x=5, y=5)$ in the Cartesian coordinates used.

The averaged streamwise velocity contours are shown in Fig. 11. The vortical structures at the downstream locations are an instantaneous flow characteristic. They are not present in the averaged results, as the vortical structures are continuously convected downstream by the mean flow. The nonswirling case shows no significant spreading until $z=7.0$. After $z=7.0$ the Kelvin-Helmholtz instability is established causing the jet to expand in the cross-streamwise direction. Conversely, the cross-streamwise jet spreading in the swirling case occurs earlier, at around $z=3.5$ due to the effects of swirl. In Fig. 11, it is noticed that at the far downstream locations after $z=7.0$ the swirling gas jet case shows decreasing spreading compared to the swirling two-phase jet, indicating that the absence of the liquid tends to reduce the flow spreading at these particular locations. The most important feature in Fig. 11 is the capturing of the GRZ and CRZ. For the nonswirling case, the GRZ adjacent to the nozzle exit on the inner side of the annulus can be seen in Fig. 11(a). This is evident from the negative values of the streamwise velocity in this region. For the swirling case, the CRZ is shown at a slightly downstream location in Fig. 11(c). By comparing Figs. 11(a) and 11(c) it is observed that there is no formation of a GRZ in the swirling case. A CRZ is formed instead, lying between $z=1.4$ and $z=2.6$. The same phenomenon occurs in the swirling gas jet case where a CRZ is formed in between $z=1.2$ and $z=2.4$. Figures 11(b), 11(d), and 11(f) show the rotating patterns of the GRZ and CRZ for all cases. For better visualization the stream traces are plotted on top of the averaged streamwise velocity contours. The stream traces clearly show the negative streamwise velocity present and they indicate the recirculating behavior of both the GRZ and the CRZ. The CRZ in the swirling gas-liquid case appears to be located slightly downstream compared to the CRZ of the swirling gas jet case.

Figure 12 shows a schematic of the recirculation zones in an annular swirling jet where the shaded part shows regions of interaction between the GRZ and CRZ. The CRZ formation is purely due to the swirling mechanism. In swirling jet flows the GRZ and the CRZ may coexist, or they may blend together, or only the CRZ may be present with the GRZ completely vanishing. ${ }^{5,50,54}$ This depends on the various inlet parameters. In an annular configuration, the GRZ adjacent to the nozzle exit is associated with the formation of a stagnation region when the jet column meets the center line. As shown in Fig. 12, a stronger CRZ may overwhelm the GRZ, and thus the interaction between the GRZ and the CRZ produces a canceling effect which completely eliminates the GRZ.

In Figs. 13(a) and 13(b), the averaged liquid volume fraction profiles at different streamwise planes are shown for both nonswirling and swirling gas-liquid cases. In Fig. 13(a) it is clear that at $z=2.0$ and $z=4.0$ the liquid volume fraction profiles show similar shapes, with one large branch and a deep crest. This is due to the annular nozzle configuration. For the nonswirling case, at $z=4.0$, the branch maximum shows a decrease from a value of $0.55($ at $z=2.0)$ to a value of 0.45 , while the crest minimum at $r=0$ is increased from 0.1 to 0.25 . This trend shows the tendency of the jet to converge toward the center line and eventually collapse. At $z=6.0$ a "top-hat-type" liquid volume fraction profile is formed, indicating that the annular column has collapsed in the cross-streamwise direction. The liquid dispersion is significantly increased at $z=8.0$. The irregular pattern of the liquid distribution is due to the complex vortical flow at this particular location. The swirling gas-liquid case shows different liquid distribution trends. At $z=2.0$, apart from the spike due to the annular configuration, a peak is observed at $r=0$. This is due to the effects of the CRZ on the liquid distribution. Since $z=2.0$ is at the heart of the CRZ, as shown in Figs. 11(c) and 11(d), the velocity reversals associated with the CRZ tend to bring liquid from the outer side toward the inner core of the jet. This tendency is not present in the nonswirling case since the CRZ is absent. The annular liquid column has already collapsed at $z=4.0$ in the swirling gasliquid case. At progressive downstream locations the dispersion of the liquid increases. As expected, in both cases, with an increasing cross-streamwise liquid dispersion, the liquid volume fraction magnitudes show a decreasing trend. The swirling motion initiates earlier the collapsing of the annular liquid column into disorganized patterns while the liquid cross-streamwise spreading is significantly increased.

Figures 13(c) and 13(d) show the spatially and temporally averaged streamwise velocity profiles in various streamwise planes in the nonswirling and swirling gas-liquid cases, respectively. The streamwise velocity in the nonswirling case shows similar trends to the averaged liquid volume fraction distributions shown in Fig. 13(a). For the swirling case, the collapsing of the jet occurs earlier and the velocity profiles are more complex than the nonswirling case. The negative velocity values at $z=2.0$ in Fig. 13(d), near the jet center line $r=0$, are due to the presence of the CRZ in this region. These velocity reversals are responsible for the small amounts of liquid dragged from the outer side toward the center location of the jet, as shown in Fig. 13(b). Both cases show decreasing velocity magnitudes at progressive downstream locations due to the mixing of the annular jet with its ambient environment. 


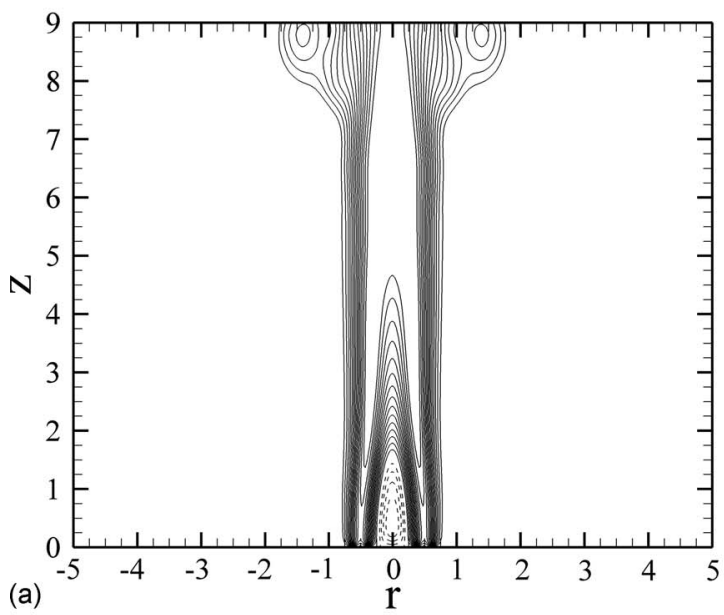

(b)
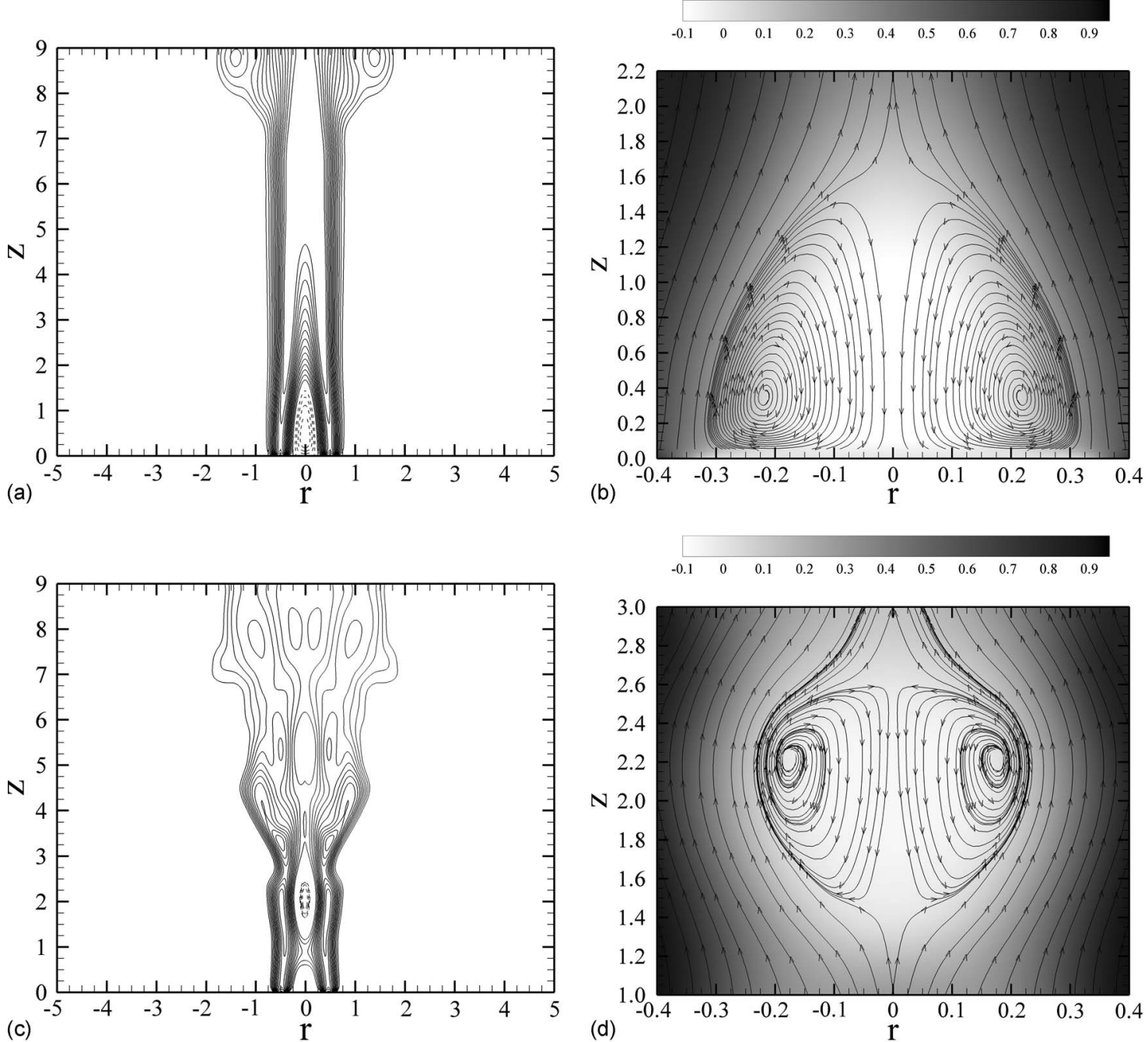

(d)
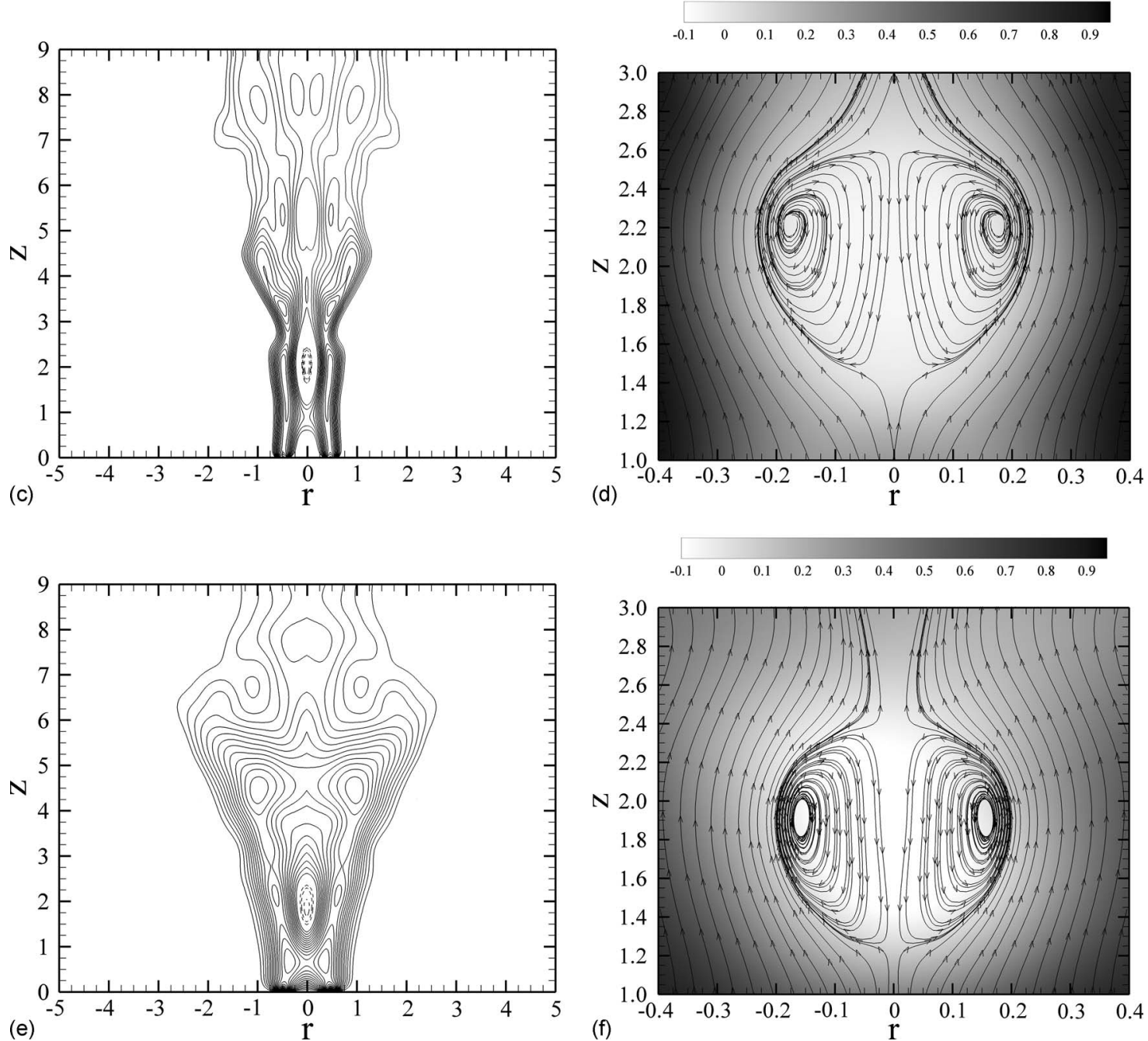

FIG. 11. Spatially and temporally averaged streamwise velocity contours and stream traces of the recirculation zones (solid line: positive; dashed line: negative). (a) Streamwise velocity $(S=0)$; (b) GRZ stream traces $(S=0)$; (c) streamwise velocity $(S=0.4)$; (d) CRZ stream traces $(S=0.4)$; (e) streamwise velocity $(S=0.4$, gas jet); (f) CRZ stream traces $(S=0.4$, gas jet)

\section{CONCLUSIONS}

In this study, the dynamics of annular nonswirling and swirling gas-liquid two-phase jets have been examined by direct solution of the Navier-Stokes equations. The mathematical formulation of the flow system is based on an Eu- lerian approach with mixed-fluid treatment. An adapted VOF method combined with a CSF model were utilized to capture the interface dynamics. Highly accurate numerical schemes have been employed for time advancement and spatial discretization. An analytical form of equilibrium swirling inflow 


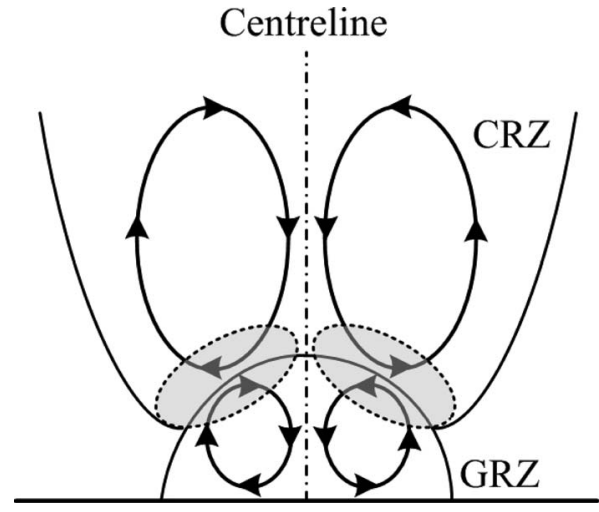

FIG. 12. Schematic of the recirculation zones in an annular swirling jet (GRZ and CRZ).

conditions has been used to generate the desired swirl level at the nozzle exit. Results from three computational cases have been presented in order to examine the effect of swirl on the flow physics and scrutinize the effect of liquid sheet on the PVC and GRZ/CRZ developments.

In both gas-liquid cases the flow becomes more vortical at downstream locations. Unsteady vortical flow characteristics are observed after $z=6.0$ for the nonswirling case while the swirling case shows dynamic structures after around $z=5.0$. The swirling motion promotes the instability and thus the liquid spatial dispersion/spreading. It was identified that swirl enhances mixing, resulting in a more homogeneous
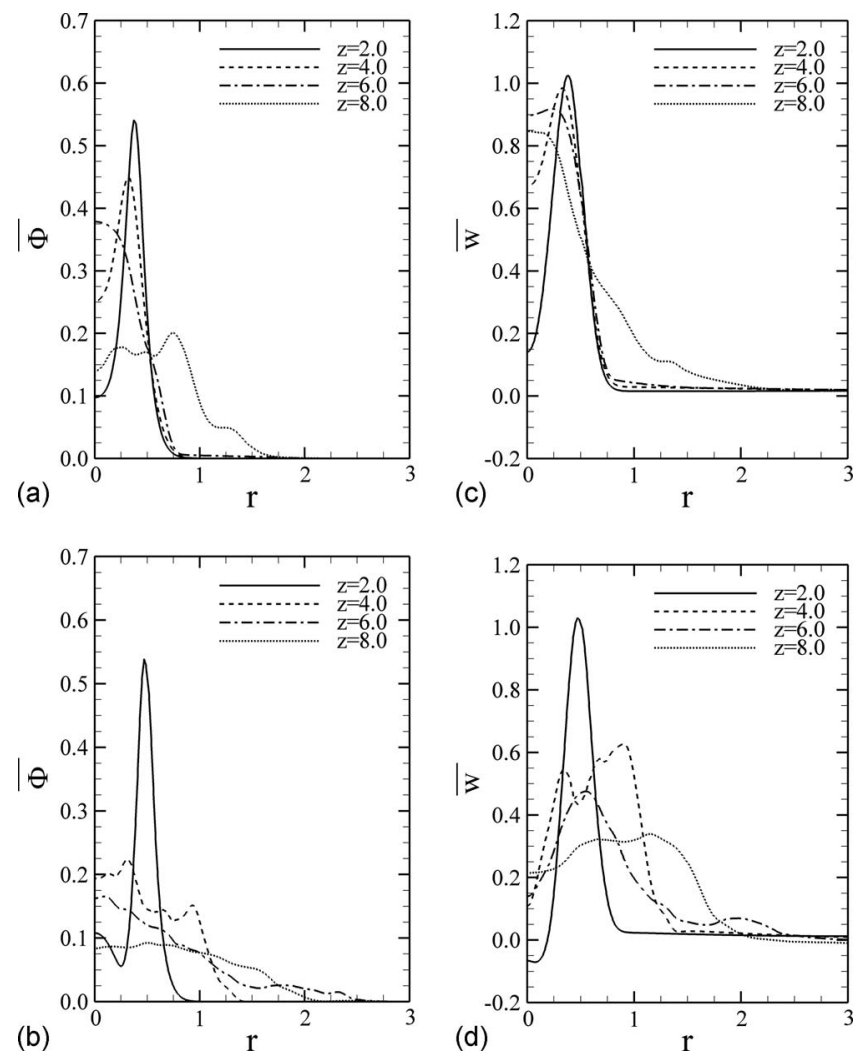

FIG. 13. Spatially and temporally averaged liquid volume fraction and streamwise velocity profiles in different streamwise planes. (a) Liquid volume fraction $(S=0)$; (b) liquid volume fraction $(S=0.4)$; (c) streamwise velocity $(S=0)$; (d) streamwise velocity $(S=0.4)$. vortical flow field at further downstream locations. The simulations have shown that the annular gas-liquid twophase jet is characterized by the formation of a GRZ next to the jet nozzle exit due to the presence of two adjacent shear layers. The addition of swirl causes the jet to develop a CRZ, at slightly upstream locations. The interactions between the GRZ and the CRZ can lead to a canceling effect, where the GRZ may completely vanish. The recirculation zones are more or less stationary while vortical structures due to the Kelvin-Helmholtz instability are dynamic. The DNS results indicate the formation of a PVC in the swirling gas-liquid case, showing that the PVC can develop in annular gasliquid two-phase jet flows at relatively low swirl number. No development of a PVC is found in the swirling gas jet case, indicating that in annular two-phase swirling jet flows, the liquid phase can play a significant role in the PVC growth, apart from other factors such as the swirl number and/or the nozzle geometry. The swirl number alone is an insufficient criterion for PVC development in such flows. Finally, high Reynolds number flows and larger computational domains need to be considered in the future to investigate the further disintegration of the liquid in a gas-liquid two-phase flow environment. However, excessive requirements for computational resources may prove to be prohibitive.

\section{ACKNOWLEDGMENTS}

This work made use of the facilities of HPCx, U.K.'s national high-performance computing service. Computing resources were provided by the UK Turbulence Consortium (UKTC) under EPSRC Grant No. EP/D044073/1.

${ }^{1}$ C. J. Choi and S. Y. Lee, "Droplet formation from a thin hollow liquid jet with a core air flow," Atomization Sprays 15, 469 (2005).

${ }^{2}$ E. A. Ibrahim and T. R. McKinney, “"Injection characteristics of nonswirling and swirling annular liquid sheets," Proc. Inst. Mech. Eng., Part C: J. Mech. Eng. Sci. 220, 203 (2006).

${ }^{3}$ J. C. Lasheras, E. Villermaux, and E. J. Hopfinger, "Break-up and atomization of a round water jet by a high-speed annular air jet," J. Fluid Mech. 357, 351 (1998).

${ }^{4}$ K. Gupta, D. G. Lilley, and N. Syred, Swirl Flows (Abacus, Kent, 1984).

${ }^{5}$ H. J. Sheen, W. J. Chen, and S. Y. Jeng, "Recirculation zones of unconfined and confined annular swirling jets," AIAA J. 34, 572 (1996).

${ }^{6}$ A. W. Hübner, M. J. Tummers, K. Hanjalíc, and Th. H. van der Meer, "Experiments on a rotating-pipe swirl burner," Exp. Therm. Fluid Sci. 27, 481 (2003).

${ }^{7}$ Y. Liao, S. M. Jeng, M. A. Jog, and M. A. Benjamin, "Instability of an annular liquid sheet surrounded by swirling airstreams," AIAA J. 38, 453 (2000).

${ }^{8}$ M. Adzic, I. S. Carvalho, and M. V. Heitor, "Visualisation of the disintegration of an annular liquid sheet in a coaxial airblast injector at low atomising air velocities," Opt. Diagnost. Eng. 5, 27 (2001).

${ }^{9} \mathrm{~K}$. Ramamurthi and T. J. Tharakan, "Flow transition in swirled liquid sheets," AIAA J. 36, 420 (1998).

${ }^{10}$ M. Sommerfeld and H.-H. Qiu, "Detailed measurements in a swirling particulate two-phase flow by a phase-Doppler anemometer," Int. J. Heat Fluid Flow 12, 20 (1991).

${ }^{11} \mathrm{M}$. Vanierschot and E. van den Bulck, "Hysteresis in flow patterns in annular swirling jets," Exp. Therm. Fluid Sci. 31, 513 (2007).

${ }^{12} \mathrm{~S}$. G. Chuech, "Numerical simulation of nonswirling and swirling annular liquid jets," AIAA J. 31, 1022 (1993).

${ }^{13}$ S. Jakirlić, K. Hanjalić, and C. Tropea, "Modeling rotating and swirling turbulent flows: A perpetual challenge," AIAA J. 40, 1984 (2002).

${ }^{14}$ M. García-Villalba, J. Fröhlich, and W. Rodi, "Identification and analysis of coherent structures in the near field of a turbulent unconfined annular swirling jet using large eddy simulation," Phys. Fluids 18, 055103 (2006). 
${ }^{15}$ M. García-Villalba and J. Fröhlich, "LES of a free annular swirling jet: Dependence of coherent structures on a pilot jet and the level of swirl," Int. J. Heat Fluid Flow 27, 911 (2006).

${ }^{16}$ M. R. Ruith and E. Meiburg, "Direct numerical simulation of spatially developing, three-dimensional swirling jets," J. Turbul. 3, N65 (2002).

${ }^{17}$ W. Kollmann, A. S. H. Ooi, M. S. Chong, and J. Soria, "Direct numerical simulations of vortex breakdown in swirling jets," J. Turbul. 2, N5 (2001).

${ }^{18}$ C. D. Pierce and P. Moin, "Large eddy simulation of a confined coaxial jet with swirl and heat release," AIAA Paper No. 98-2892, 29th Fluid Dynamics Conference, Albuquerque, NM, 15-18 June 1998.

${ }^{19}$ P. Wang, X. S. Bai, M. Wessman, and J. Klingmann, "Large eddy simulation and experimental studies of a confined turbulent swirling flow," Phys. Fluids 16, 3306 (2004).

${ }^{20}$ X. Lu, S. Wang, H.-G. Sung, S.-Y. Hsieh, and V. Yang, "Large eddy simulations of turbulent swirling flows injected into a dump chamber," J. Fluid Mech. 527, 171 (2005).

${ }^{21}$ B. Wegner, A. Maltsev, C. Schneider, A. Sadiki, A. Dreizler, and J. Janicka, "Assessment of unsteady RANS in predicting swirl flow instability based on LES and experiments," Int. J. Heat Fluid Flow 25, 528 (2004).

${ }^{22}$ B. Wegner, A. Kempf, C. Schneider, A. Sadiki, A. Dreizler, J. Janicka, and M. Schäfer, "Large eddy simulation of combustion processes under gas turbine conditions," Prog. Comput. Fluid Dyn. 4, 257 (2004).

${ }^{23}$ S. Banerjee, D. Lakehal, and M. Fulgosi, "Surface divergence models for scalar exchange between turbulent streams," Int. J. Multiphase Flow 30, 963 (2004).

${ }^{24}$ M. Fulgosi, D. Lakehal, S. Banerjee, and V. De Angelis, "Direct numerical simulation of turbulence in a sheared air-water flow with a deformable interface," J. Fluid Mech. 482, 319 (2003).

${ }^{25}$ P. Lombardi, V. De Angelis, and S. Banerjee, "Direct numerical simulation of near-interface turbulence in coupled gas-liquid flow," Phys. Fluids 8 , 1643 (1996)

${ }^{26} \mathrm{M}$. Klein, "Direct numerical simulation of a spatially developing water sheet at moderate Reynolds number," Int. J. Heat Fluid Flow 26, 722 (2005).

${ }^{27}$ G. A. Siamas, X. Jiang, and L. C. Wrobel, "A numerical study of an annular liquid jet in a compressible gas medium," Int. J. Multiphase Flow 34, 393 (2008).

${ }^{28}$ G. A. Siamas and X. Jiang, "Direct numerical simulation of a liquid sheet in a compressible gas stream in axisymmetric and planar configurations," Theor. Comput. Fluid Dyn. 21, 447 (2007).

${ }^{29}$ X. Jiang and G. A. Siamas, "Direct computation of an annular liquid jet," J. Algo. Comput. Tech.1748-3018 1, 103 (2007).

${ }^{30}$ C. T. Crowe, Multiphase Flow Handbook (Taylor \& Francis, New York, 2006)

${ }^{31}$ C. W. Hirt and B. D. Nichols, "Volume of fluid (VOF) method for the dynamics of free boundaries," J. Comput. Phys. 39, 201 (1981).

${ }^{32}$ D. Gueyffier, J. Li, A. Nadim, R. Scardovelli, and S. Zaleski, "Volumeof-fluid interface tracking with smoothed surface stress methods for threedimensional flows," J. Comput. Phys. 152, 423 (1999).

${ }^{33}$ J. U. Brackbill, D. B. Kothe, and C. Zemach, "A continuum method for modelling surface tension,” J. Comput. Phys. 100, 335 (1992).
${ }^{34}$ J. H. Williamson, "Low-storage Runge-Kutta schemes," J. Comput. Phys. 35, 48 (1980)

${ }^{35}$ S. K. Lele, "Compact finite-difference schemes with spectral like resolution," J. Comput. Phys. 103, 16 (1992).

${ }^{36} \mathrm{~K}$. W. Thompson, "Time dependent boundary conditions for hyperbolic systems," J. Comput. Phys. 68, 1 (1987).

${ }^{37} \mathrm{X}$. Jiang and K. H. Luo, "Direct numerical simulation of the puffing phenomenon of an axisymmetric thermal plume," Theor. Comput. Fluid Dyn. 14, 55 (2000).

${ }^{38}$ B. E. Mitchell, S. K. Lele, and P. Moin, "Direct computation of the sound generated by vortex pairing in an axisymmetric jet," J. Fluid Mech. 383, 113 (1999).

${ }^{39}$ C. D. Pierce and P. Moin, "Method for generating equilibrium swirling inflow conditions," AIAA J. 36, 1325 (1998).

${ }^{40}$ X. Jiang, G. A. Siamas, and L. C. Wrobel, "Analytical equilibrium swirling inflow conditions for computational fluid dynamics," AIAA J. 46, 1015 (2008).

${ }^{41}$ T. Uchiyama, "Three-dimensional vortex simulation of bubble dispersion in excited round jet," Chem. Eng. Sci. 59, 1403 (2004).

${ }^{42}$ A. K. M. F. Hussain and K. B. M. Q. Zaman, "The preferred mode of the axisymmetric jet," J. Fluid Mech. 110, 39 (1981).

${ }^{43}$ R. H. Perry and D. W. Green, Perry's Chemical Engineers' Handbook, 7th ed. (McGraw-Hill, Singapore, 1998).

${ }^{44}$ S. B. Pope, Turbulent Flows (Cambridge University Press, Cambridge, 2000).

${ }^{45}$ M. Freitag and M. Klein, "Direct numerical simulation of a recirculating, swirling flow," Flow, Turbul. Combust. 75, 51 (2005).

${ }^{46}$ S. Baik, J. P. Blanchard, and M. L. Corradini, "Development of microdiesel injector nozzles via microelectromechanical systems technology and effects on spray characteristics," ASME J. Eng. Gas Turbines Power 125, 427 (2003).

${ }^{47}$ M. R. Wang and Z. X. Li, "Numerical simulations on performance of MEMS-based nozzles at moderate or low temperatures," Microfluid. Nanofluid. 1, 62 (2004).

${ }^{48}$ X. Jiang, H. Zhao, and K. H. Luo, "Direct computation of perturbed impinging hot jets," Comput. Fluids 36, 259 (2007).

${ }^{49} \mathrm{X}$. Jiang and K. H. Luo, "Dynamics and structure of transitional buoyant jet diffusion flames with sidewall effects," Combust. Flame 133, 29 (2003).

${ }^{50} \mathrm{~N}$. Syred, "A review of oscillation mechanisms and the role of the precessing vortex core (PVC) in swirl combustion systems," Prog. Energy Combust. Sci. 32, 93 (2006).

${ }^{51}$ Y. M. Al-Abdeli and A. R. Masri, "Recirculation and flowfield regimes of unconfined non-reacting swirling flows," Exp. Therm. Fluid Sci. 27, 655 (2003).

${ }^{52}$ Y. M. Al-Abdeli and A. R. Masri, "Precession and recirculation in turbulent swirling isothermal jets," Combust. Sci. Technol. 176, 645 (2004).

${ }^{53} \mathrm{M}$. Vanierschot and E. van den Bulck, "Influence of the nozzle geometry on the hysterisis of annular swirling jets," Combust. Sci. Technol. 179, 1451 (2007).

${ }^{54}$ O. Lucca-Negro and T. O’Doherty, "Vortex breakdown: a review," Prog. Energy Combust. Sci. 27, 431 (2001). 